\title{
New erbium-doped fiber laser with a double tunable bandpass filter
}

\author{
Ali Sellami \\ Khalid al-Khateeb \\ International Islamic University \\ Faculty of Engineering \\ Electrical and Computer Engineering \\ Department \\ Malaysia \\ E-mail: sellami2003@ hotmail.com
}

\section{Bouzid Billoui}

Hafr Al-batin Community College (HBCC)

Saudi Arabia

\begin{abstract}
A new configuration for an erbium-doped fiber laser is reported. It incorporates a linear cavity with fiber loopback and a tunable bandpass filter (TBF). This configuration suppresses the amplified spontaneous emission and achieves a highly stable output power of more than $18 \mathrm{dBm}$ at $1560 \mathrm{~nm}$. A standard spectrum is obtained with TBF adjustment. () 2008 Society of Photo-Optical Instrumentation Engineers. [DOl: 10.1117/1.2909668]
\end{abstract}

Subject terms: erbium; fiber; laser; TBF; optical communications.

Paper 070051R received Jan. 25, 2007; revised manuscript received Dec. 17, 2007; accepted for publication Jan. 28, 2008; published online May 1, 2008.

\section{Introduction}

Tunable single-frequency lasers in the wavelength region, around $1550 \mathrm{~nm}$ are of much interest in a variety of applications, such as wavelength division multiplexing (WDM) optical communications, spectroscopy, and fiber sensors. ${ }^{1,2}$ These lasers have potential advantages because of their narrow line width and low-intensity noise. They represent a natural source for fiber optical communications, since the light is already in the fiber and they can be spliced directly to the system. Other advantages include high sidemode suppression ratio (SMSR), low threshold, and flat output power. These parameters are important in the design considerations for this type of lasers.

Various configurations have been proposed aimed at achieving best combination of these characteristics. They include ring cavity ${ }^{3}$ and linear cavity ${ }^{4}$ structures. The suggested tuning range in the conventional band ( $\mathrm{C}$ band) and long band (L band) is reported to be $80 \mathrm{~nm} .{ }^{5}$ The placement of the fused coupler for tapping the output from the system is critical due to the presence of amplified spontaneous emission (ASE) in the laser output. For efficient ASE filtering, the fused coupler is placed after the tunable filter. ${ }^{6}$ Inefficient filtering can lead to the deterioration of the SMSR value, which is critical for WDM transmission.

In this paper, we propose a new linear cavity erbiumdoped fiber laser (EDFI) configuration using a fiber loopback (FLB) embedded with tunable filters in a linear laser oscillator. Implementing the linear cavity configuration, three circulators and two tunable bandpass filters (TBFs) are used. The output power of the proposed laser design is more than $18 \mathrm{dBm}$ at $1560 \mathrm{~nm}$. The double filtering technique enables the laser configuration to achieve higher stability as well as higher output power.

\section{Experimental Setup}

Figure 1 shows the proposed configuration of the system. It consists of three circulators: CIR1 with four ports, and CIR2 and CIR3 with three ports each. They are used as loopback mirrors. The arrangement allows the insertion of

0091-3286/2008/\$25.00 @ 2008 SPIE the output coupler at one of the FLB mirrors. The TBF (Newport) is mechanically tuned with a passband of $1 \mathrm{~nm}$, an insertion loss of around $1.5 \mathrm{~dB}$ at the center wavelength, and a tuning range limited to $40 \mathrm{~nm}$, viz., from 1525 to $1565 \mathrm{~nm}$. Both TBFs must be tuned and adjusted to the same wavelength in order to suppress the ASE efficiently. The tuning process is used to stabilize the output power, to control the shape of the spectrum, and to minimize the insertion loss.

The linear laser cavity consists of two portions. The first, EDF1, is a 10-m length, and the second, EDF2, is a 15-m length, both of erbium-doped fiber, with an $\mathrm{Er}^{3+}$ ion concentration of $440 \mathrm{ppm}$. The EDF is pumped by two $980-\mathrm{nm}$ laser diodes. Each diode can provide a pump power of $220 \mathrm{~mW}$ (max). A fused coupler with $95 \%$ coupling ratio is placed after the tunable filter in one of the fiber loop mirrors to act as the output port. All connections are fusionspliced to minimize any backreflection and to achieve a low cavity loss. An optical spectrum analyzer (OSA) with $0.01-\mathrm{nm}(\mathrm{min})$ resolution constituted the principal part of the measuring equipment.

\section{Results and Discussion}

The effect of pump power on the performance of the laser output was investigated by varying the power of the pump, which was provided by two $980-\mathrm{nm}$ laser diodes. The power of the first pump $\left(P_{1}\right)$ is fixed at $10 \mathrm{~mW}$, and then the power of second pump $\left(P_{2}\right)$ is varied from 10 to $220 \mathrm{~mW}$ in steps of $10 \mathrm{~mW}$. When $P_{2}$ reaches $220 \mathrm{~mW}, P_{1}$ is raised similarly in steps of $10 \mathrm{~mW}$ until it reaches $220 \mathrm{~mW}$ also. For a selected lasing wavelength of $1560 \mathrm{~nm}$, the output power of the laser system is found to increase with increasing pump power until it becomes $18 \mathrm{dBm}$, with a coupling ratio of $95 \%$. Figure 2 illustrates the lasing output power versus the pumping power from the two diodes, which was obtained by fixing $P_{2}$ at 50,100 , 150 , and $220 \mathrm{~mW}$.

Figure 3 depicts the optical spectra of the output, using the OSA with a $0.1-\mathrm{nm}$ resolution. If the two pumps are operated at $220 \mathrm{~mW}$ each with output coupling of $95 \%$, the laser output power at the 1560 -nm wavelength becomes 


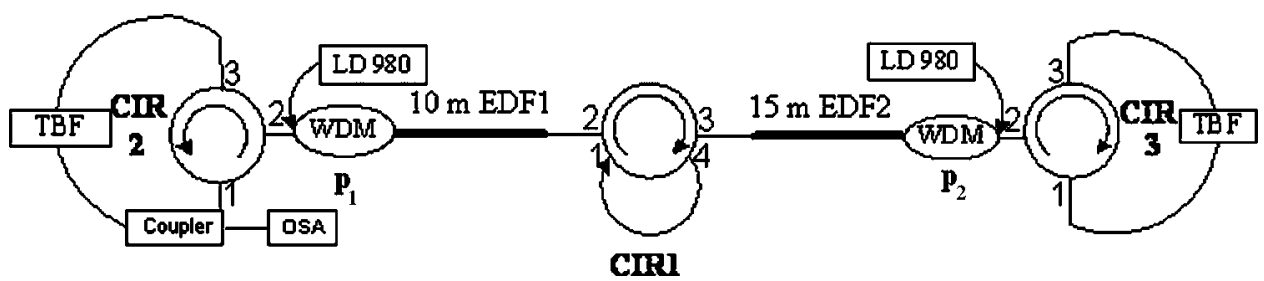

Fig. 1 Configuration of EDFL with fiber loop mirrors embedded with a tunable bandpass filter.

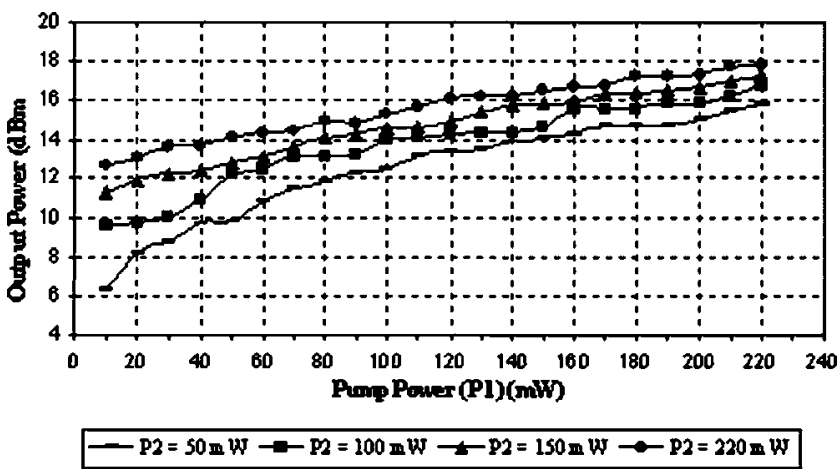

Fig. 2 Output power at 1560-nm lasing wavelength versus pump power from 980-nm laser diodes.
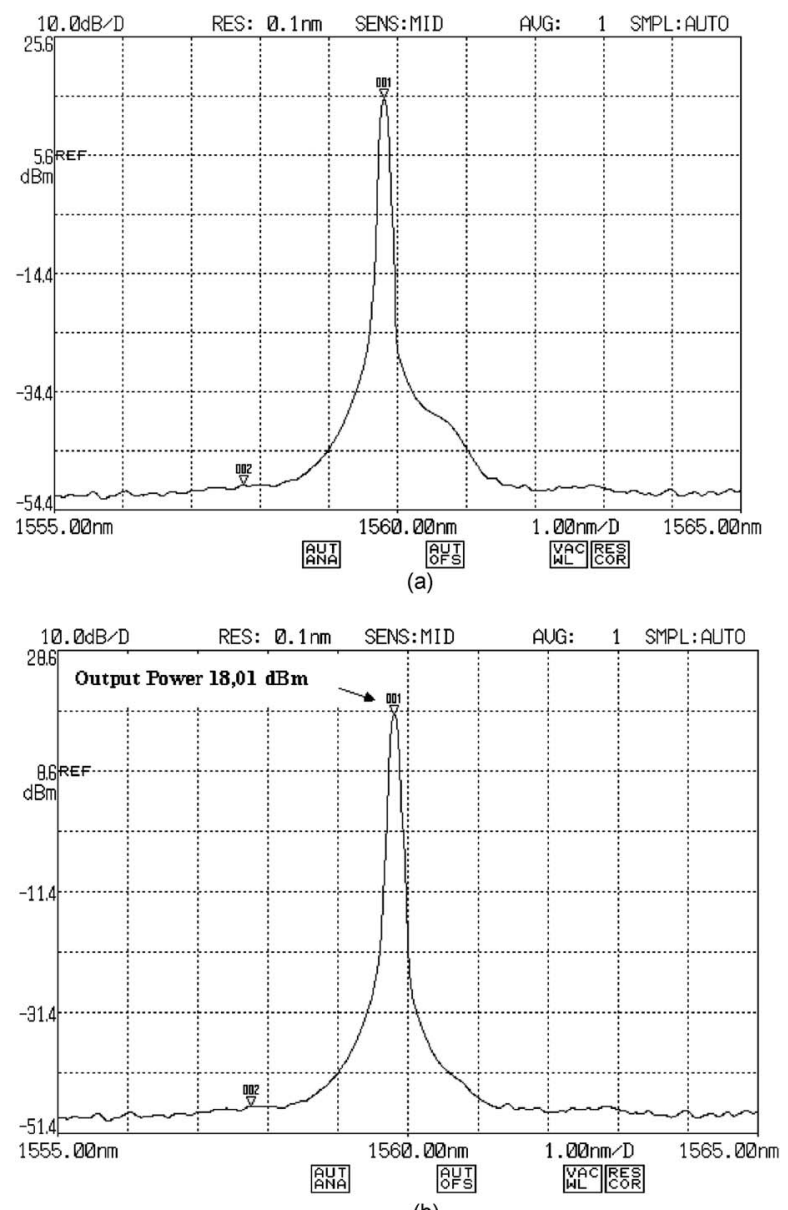

Fig. 3 Output spectra of the proposed laser system at $1560 \mathrm{~nm}$ (OSA resolution bandwidth of $0.1 \mathrm{~nm}$ ): (a) before adjustment, (b) after adjustment.
$18 \mathrm{~mW}$, with a 64-dB SMSR. The noise level of the ASE between 1555 and $1565 \mathrm{~nm}$ is flat (below the detection level of the OSA). This indicates the effectiveness of the double filtering technique employed in this design. Therefore, the SMSR value of the laser can be maintained (higher than $64 \mathrm{~dB}$ ) over the tuning range.

Figure 3(a) shows a deformation at the bottom right of the output spectrum, which was made to disappear by adjustments of the two TBFs. These adjustments were made by mechanical tuning of the two TBFs alternately until the right shape was obtained, as shown in Fig. 3(b).

It has been observed that the location and the adjustment

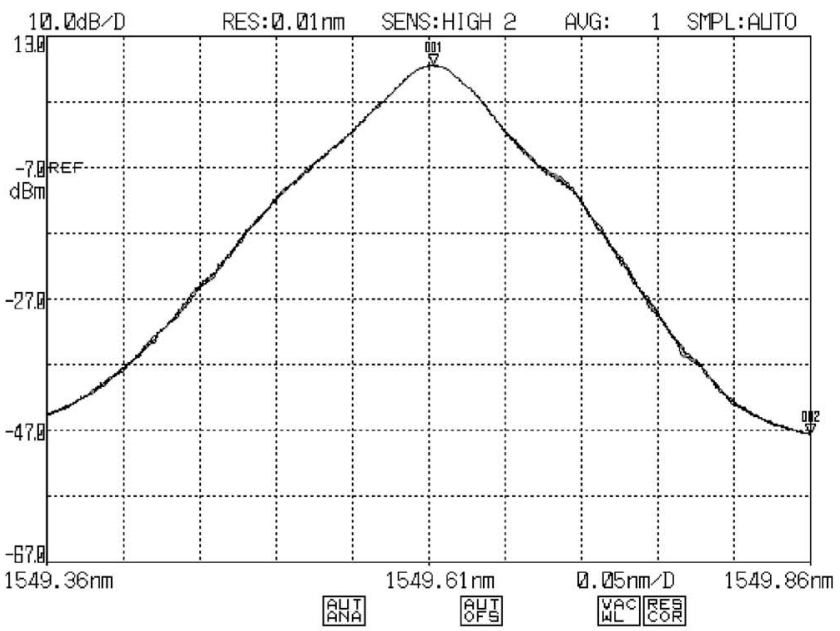

(a)

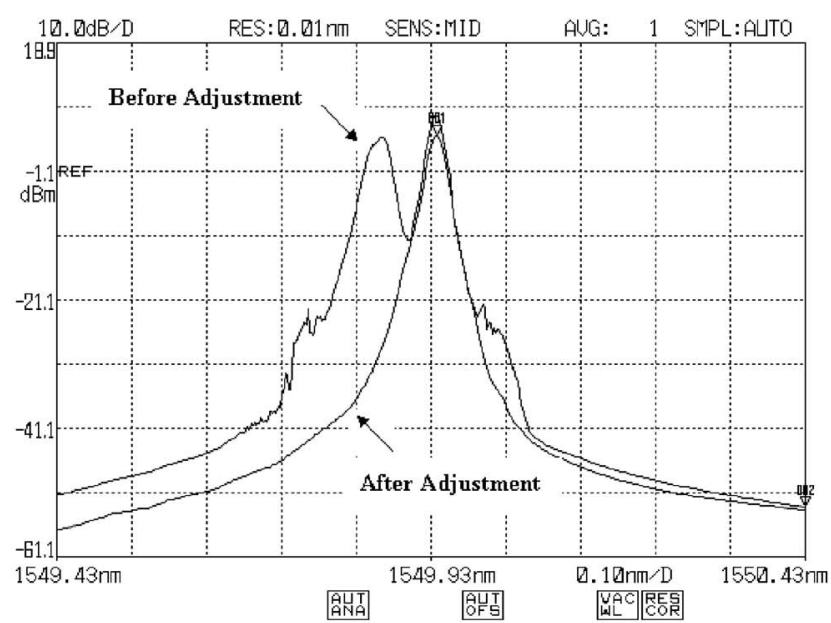

(b)

Fig. 4 (a) The stability of output optical spectra. (b) Controlling the shape of the output lasing spectra. 
of the two TBFs in this configuration have an intrinsic effect on ASE suppression and spectrum shape control, and play an essential role in the stability of the signal. It was mentioned in a previous work $^{7}$ that the shape of the laser signal cannot be controlled or changed. In this work, however, it has been demonstrated that with good filtering of the ASE by placing the TBF before the output coupler leads, and with proper adjustments, good performance can be attained, as shown in Fig. 3. The four overlap spectra of lasing power with $0.01-\mathrm{nm}$ resolution and 0.5-nm span, depicted in Fig. 4(a), prove that good stability of output optical spectra can be achieved with the proposed laser system. Figure 4(b) shows the two spectra before and after the adjustment of the two TBFs.

\section{Conclusion}

A new EDFL architecture with high output lasing power of $18 \mathrm{dBm}$, high stability, and high SMSR of $64 \mathrm{~dB}$ has been realized. Efficient noise suppression in a linear cavity is successfully demonstrated utilizing fiber loop mirrors. Two TBFs in the design played an essential role in the stability and shape control of the output lasing spectrum, so that a standard shape could be obtained.

\section{References}

1. P. L. Scrivener, E. J. Tarbox, and P. D. Maton, "Narrow linewidth tunable operation of $\mathrm{Er}^{3+}$ doped single-mode fiber laser," Electron. Lett. 25, 549-550 (1989).

2. T. Pfeiffer, H. Schmuck, and H. Blow, "Output power characteristics of erbium-doped fiber ring laser," IEEE Photonics Technol. Lett. 4, 847-849 (1992).
3. A. Bellemare, M. Karasek, C. Riviere, F. Babin, G. He, V. Roy, and G. W. Schinn, "A broadly tunable erbium-doped fiber ring laser: experimentation and modeling," IEEE J. Sel. Top. Quantum Electron. 7 22-29 (2001).

4. H. Chen, F. Babin, M. Leblanc, and G. W. Schinn, "Widely tunable single-frequency Er-doped fiber laser with long linear cavity," IEEE Photonics Technol. Lett. 13(4), 287-289 (2001).

5. S. Yamashita and M. Nishihara, "Widely tunable erbium-doped fiber ring laser covering both C-band and L-band," IEEE J. Sel. Top. Quantum Electron. 7, 41-43 (2001).

6. H. Chen, F. Babin, M. Leblanc, and G. W. Schinn, "Widely tunable single-frequency erbium-doped fiber lasers," IEEE Photonics Technol. Lett. 15(2), 185-187 (2003).

7. X. Dong, N. Q. Ngo, P. Shum, H. Y. Tam, and X. Dong, "Linear cavity erbium-doped fiber laser with over $100 \mathrm{~nm}$ tuning range," Opt. Express 11, 1689-1664 (2003).

Ali Sellami graduated from Algeria University. He worked at the University of Biskra (Algeria) as a computer lecturer and joined Textile and Enterprise Equipment in Biskra, as an electrical engineer. $\mathrm{He}$ received his master's degree from a Malaysian, university, where he worked on design and analysis of a new erbium-doped fiber amplifier and laser. He is currently pursuing his PhD in quantum cryptography at the International Islamic University, Malaysia.

Khalid al-Khateeb received his BSc at the Royal College of Advanced Technology, UK, in 1966, MSc at the University of Salford, UK, in 1971, and PhD at the University of Manchester in 1975. He has held many academic posts at various Middle East universities. Professor Al-Khateeb has been at the International Islamic University, Malaysia, since July 2000.

Bouzid Billoui graduated from Setif University. He received his master's degree from Malay University and his PhD degree from UPM. He worked at Multi-media University in Malaysia as a lecturer. Currently he is a lecturer at Hafr Al-batin Community College (HBCC), Saudi Arabia. 